\title{
Polarized parton distribution functions: parametrization and transverse momentum dependence
}

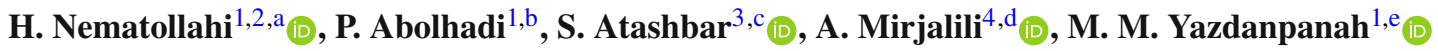 \\ ${ }^{1}$ Faculty of Physics, Shahid Bahonar University of Kerman, Kerman, Iran \\ ${ }^{2}$ Mahani Mathematical Research Center, Shahid Bahonar university of Kerman, Kerman, Iran \\ ${ }^{3}$ School of Particles and Accelerators, Institute for Research in Fundamental Sciences (IPM), P.O.Box 19395-5531, Tehran, Iran \\ ${ }^{4}$ Physics Department, Yazd University, P.O.Box 89195-741, Yazd, Iran
}

Received: 7 October 2020 / Accepted: 28 December 2020 / Published online: 12 January 2021

(C) The Author(s) 2021

\begin{abstract}
We extract the polarized parton distribution functions (PPDFs) of proton. To this end we do a global analysis on recent and up-to-dated experimental data of polarized nucleon and deuteron structure functions at the NLO accuracy of perturbative QCD. We use the Jacobi polynomials expansion technique to do the required analyses. To achieve a more realistic estimation of the PPDFs and observable uncertainties we employ the Hessian error propagation. Following that we apply a theoretical approach which is based on imposing Lorentz structure in the parton model to investigate the transverse momentum dependence of these PPDFs. In this approach, the transverse momentum dependent (TMD) polarized parton distributions, which are also called the helicity distributions, are related to the integrated ones. This relation is indicating the priority and importance of data analysis for PPDFs. The results show a convenient general treatment expected for TMD densities which are in fact resulted from data analysis and fitting processes of integrated PPDFs in an adequate and a proper way.
\end{abstract}

\section{Introduction}

The parton distribution functions (PDFs) are related to observable like the cross section in deep inelastic scattering processes, electron-positron annihilation and proton-proton collisions. QCD analysis of deep-inelastic scattering (DIS) data provides us new insight into the hadron physics. The reliability of theoretical results can be increased by testing

\footnotetext{
a e-mail: hnematollahi@uk.ac.ir (corresponding author)

b e-mail: p_abolhadi@phy.uk.ac.ir

c e-mail: Atashbar@ipm.ir

de-mail: A.Mirjalili@yazd.ac.ir

e e-mail: myazdan@uk.ac.ir
}

the analysis, for instance, of a hard lepton scattering off the hadrons. A reliable description of DIS at large momentum transfer can be obtained, considering the twist expansion and factorization theorem [1]. Following that, one can use the DIS processes to better understand the nucleon structure function. In this regard, for instance the polarized structure functions can be first modeled, using different parametrization methods. Then they can be evolved to high-energy scales, employing the QCD evolution equations [2-5]. During the evolution process, the numerical values of structure functions are affected by the energy scale, at which the renormalized coupling constant as the perturbation expansion parameter is evaluated. On the other hand, the analysis of the structure functions should lead to consistent results with the related experimental data. A further step is the evolution of these densities to high-energy scales. Now the concentration is on the nature of the short-distance structure of polarized nucleons which is one of the central questions of present day hadron physics. For several years, polarized inclusive deep inelastic scattering has been the main source of information on how the individual partons in the nucleon are polarized at very short distances [6,7]. As it can be seen the data analysis and the way to fit the available data to extract the initial polarized PDFs is an important ingredient. One of the important parton distributions, regarded to study the polarized structure of nucleon, is related to the longitudinal polarized quark inside longitudinal polarized nucleon. This distribution is denoted by $\Delta q(x)$ at fixed $Q^{2}$ scale in this article. We extract the polarized parton distributions by global analysing the available experimental data of polarized hadron structure function $g_{1}\left(x, Q^{2}\right)$.

In exact consideration of inclusive DIS processes it is required to take into account the distributions in which the role of transverse momentum is embedded. These distributions are called transverse momentum dependent parton dis- 
tribution functions (TMDs) [8-16]. TMDs are the generalization of PDFs which provide us an extensive knowledge to investigate the hadron structure function. In fact the concept of PDFs can be extended by bringing the role of the intrinsic motion of partons into the TMDs in which the longitudinal and transverse momentum are considered together.

On the other hand the DIS processes are sensitive to longitudinal degree of freedom and just provide us some information for PDFs and noting say about TMDs. To achieve the three dimensional (3D) picture of nucleon, some processes like semi inclusive DIS (SIDIS) [17] are required in which one can measure the effect of transverse momentum in produced hadron. The internal structure and parton correlations of the nucleon can also be manifested, considering the spin structure of transverse nucleon. We probe the transverse momentum dependence of the polarized quark distribution $\Delta q$ in this article.

Many experiments in transverse spin measurements and also theoretical investigations have been performed increasingly in recent years. These measurements include Relativistic Heavy Ion Collider (RHIC) at Brookhaven National Laboratory (BNL). In fact measurement of large single spin asymmetries (SSAs) in pp scattering has been done by RHIC in the forward region of the polarized proton beam by the BRAHMS, PHENIX, and STAR experiments for the charged and neutral hadrons [18-24]. Large SSAs in SIDIS processes of electrons or muons off transversely polarized nucleon targets have additionally been observed in high-energy lepton scattering experiments, including HERMES at DESY, COMPASS at CERN, and several experiments at Thomas Jefferson National Accelerator Facility (JLab) [25-37].

One of the important models, which has been used to investigate the 3D picture of hadrons in both unpolarized and polarized cases, is the covariant parton model (CPM). The analysis in this model is based on the structure of the parton wave function in the nucleon rest frame [38-46]. Here we study the transverse momentum dependence of polarized PDFs applying a more general model which is based upon imposing Lorentz covariance in the parton model [47] whereas the obtained results are in agreement with those of CPM $[39,41,45]$.

In order to study the transverse momentum dependence for polarized PDFs in the framework of Ref. [47], it is required to analyse the available data on polarized nucleon structure function to extract PPDFs at initial energy scale. This is done in Sect. 2 where the last relevant data is analysed. A parameterized form for polarized PDFs is introduced there. Then we resort to Jacobi polynomial expansion to convert the polarized nucleon structure function from Mellin moment space to Bjorken $x$-space. The fit in $x$-space is done using the CERN program library MINUIT $[48,49]$. The uncertainties of PPDFs are determined, based on the Hessian method [50]. The parameterized model for PPDFs at initial energy scale is introduced in Sect. 2. The unknown parameters are adjusted so as to fulfill the required sum rules. Following that the polarized nucleon structure function is constructed, using the fitted results for parameterized PPDFs at the initial energy scale. The calculations of fitting processes can be done with more accuracy in Mellin moment space. The numerical results indicate the adequate of fit. On this base the technique of Jacobi polynomials transformation is applied to convert the results in Mellin moment space to Bjorken xspace. The results confirm the validation of data analysis which are utilized in this paper.

To achieve the goal which we are looking for it, this paper is organized as it follows. In Sect. 2 we extract the polarized parton distribution functions of proton by analysing the recent and last available data of nucleon and deuteron polarized structure functions. The details of fit procedure is demonstrated in Sect. 3. Then the transverse momentum dependence of these helicity distributions is investigated using a theoretical approach in Sect. 4. We present the results of TMD helicity distributions in Sect. 5. Finally the Sect. 6 is devoted to conclusion.

\section{Data analysing}

\subsection{Parametrization}

The spin dependent parton distribution functions have been investigated so far in perturbative QCD (pQCD), assuming different functional forms for them [51-63]. We utilize similar specifications as in ATMY13 [64], TKAA16 [65] and KTA17 [66] to do the required analysis for the relevant global fit. The parameterized form which is used for the polarized PDFs at the initial scale $\mathrm{Q}_{0}^{2}=1 \mathrm{GeV}^{2}$ would be:

$x \Delta q\left(x, Q_{0}^{2}\right)=\mathcal{N}_{q} \eta_{q} x^{\alpha_{q}}(1-x)^{\beta_{q}}\left(1+\gamma_{q} x\right)$.

Here $\mathcal{N}_{q}$ as the normalization factor, is determined by

$\frac{1}{\mathcal{N}_{q}}=\left(1+\gamma_{q} \frac{\alpha_{q}}{\alpha_{q}+\beta_{q}+1}\right) B\left(\alpha_{q}, \beta_{q}+1\right)$,

where $B\left(\alpha_{q}, \beta_{q}+1\right)$ is the Euler's beta function. The general symbol $\Delta q$ is corresponding to individual polarized up-valence, down-valence, sea and gluon distributions as $\Delta u_{v}, \Delta d_{v}, \Delta \bar{q}, \Delta g$. In our analysis the present data do not contain any contribution from the charm and bottom quarks. Since SIDIS data does not exist in the current analysis and it is supposed that the $\mathrm{SU}(3)$ flavor symmetry is established then the fit is done such that $\Delta \bar{q} \equiv \Delta \bar{u}=\Delta \bar{d}=\Delta \bar{s}=\Delta s$.

Taking into account the relations between the unknown parameters in Eq. (1) would help to follow the fit faster 
and more reliable. In fact two $\mathcal{N}_{q}$ and $\eta_{q}$ parameters are not independent. There is a relation between these two parameters since $\eta_{q}$ are the first moments of $\Delta q_{i}\left(x, Q_{0}^{2}\right)$, as $\eta_{i}=\int_{0}^{1} d x \Delta q_{i}\left(x, Q_{0}^{2}\right)$.

Now description of the fit is as it follows. We should note that the polarized DIS data do not include sufficient accuracy to determine the parameters precisely. Considering all parton types then Eq. (1) implies totally 14 free parameters where some of the parameters have been fixed in advanced and discarded in fit. For this purpose the axial charges for octet baryon, $F$ and $D$ measured in hyperon and neutron $\beta$ decay can be used to yield us numerical values for the first moments of polarized valence distribution. Using the relevant constrains, one obtains the values $\eta_{u_{v}}=0.928 \pm 0.014$ and $\eta_{d_{v}}=-0.342 \pm 0.018$ [67]. The first moments of the two valences are then fixed to their central values. In continuation the parameters $\eta_{\bar{q}}$ and $\eta_{g}$ are determined from the fit. To obtain a good description of data, the factor $\left(1+\gamma_{q} x\right)$ performs the required flexibility for the valence distributions which are in fact the $\left\{\gamma_{u_{v}}, \gamma_{d_{v}}\right\}$ parameters. The parameters $\gamma_{\bar{q}}$ and $\gamma_{g}$ for sea and gluon densities are fixed to zero.

After specifying the statues of the parameters, we are now at the situation to determine them in the fit. In this regard one can do the concerned computations in the Mellin moment space. The Mellin transform of structure function, $x g_{1}\left(x, Q^{2}\right)$, is given by:

$\mathcal{M}\left[x g_{1}, \mathrm{~N}\right]\left(Q^{2}\right) \equiv \int_{0}^{1} d x x^{\mathrm{N}-2} x g_{1}\left(x, Q^{2}\right)$.

In this space the moment of the polarized structure function $\mathcal{M}\left[x g_{1}^{p}, \mathrm{~N}\right]\left(Q^{2}\right)$ for proton at the twist- 2 contribution can be expressed in terms of PPDFs and the coefficient functions $\Delta C_{i}^{N}$ as it follows [68]

$$
\begin{aligned}
\mathcal{M}\left[x g_{1}^{p}, \mathrm{~N}\right]\left(Q^{2}\right)= & \frac{1}{2} \sum_{q} e_{q}^{2}\left\{\left(1+\frac{\alpha_{s}}{2 \pi} \Delta C_{q}^{N}\right)\right. \\
& \times\left[\Delta q\left(N, Q^{2}\right)+\Delta \bar{q}\left(N, Q^{2}\right)\right] \\
& \left.+\frac{\alpha_{s}}{2 \pi} 2 \Delta C_{g}^{N} \Delta g\left(N, Q^{2}\right)\right\} .
\end{aligned}
$$

In this equation the sum runs over quark flavors $\{u, d, s\}$ and $\{\Delta q, \Delta \bar{q}, \Delta g\}$ are denoting the polarized quark, antiquark, and gluon distributions in Mellin $N$-space respectively, obtained by a similar transform as Eq. (3). Finally $\Delta C_{i}^{N}$ is representing the polarized Wilson coefficients in Mellin space and are given by [68]:

$$
\begin{aligned}
\Delta C_{q}^{N}= & \frac{4}{3}\left\{-S_{2}(N)+\left(S_{1}(N)\right)^{2}+\left(\frac{3}{2}-\frac{1}{N(N+1)}\right)\right. \\
& \left.\times S_{1}(N)+\frac{1}{N^{2}}+\frac{1}{2 N}+\frac{1}{N+1}-\frac{9}{2}\right\},
\end{aligned}
$$

$\Delta C_{g}^{N}=\frac{1}{2}\left[-\frac{N-1}{N(N+1)}\left(S_{1}(N)+1\right)-\frac{1}{N^{2}}+\frac{2}{N(N+1)}\right]$

with $S_{1}(n)=\sum_{j=1}^{n} \frac{1}{j}=\psi(n+1)+\gamma_{E}, S_{2}(n)=$ $\sum_{j=1}^{n} \frac{1}{j^{2}}=\left(\frac{\pi^{2}}{6}\right)-\psi^{\prime}(n+1), \psi(n)=\Gamma^{\prime}(n) / \Gamma(n)$ and $\psi^{\prime}(n)=d^{2} \ln \Gamma(n) / d n^{2}$.

Now the moment of polarized structure function $\mathcal{M}\left[x g_{1}^{n}\right.$, $\mathrm{N}]\left(Q^{2}\right)$ at the twist-2 contribution can be written similarly for neutron in terms of the coefficient functions $\Delta C_{i}^{N}$ and PPDFs such that:

$$
\begin{aligned}
\mathcal{M}\left[x g_{1}^{n}, \mathrm{~N}\right]\left(Q^{2}\right)= & \mathcal{M}\left[x g_{1}^{p}, \mathrm{~N}\right]\left(Q^{2}\right)-\frac{1}{6}\left(1+\frac{\alpha_{s}}{2 \pi} \Delta C_{q}^{N}\right) \\
& \times\left(\Delta u_{v}\left(N, Q^{2}\right)-\Delta d_{v}\left(N, Q^{2}\right)\right) \cdot(7)
\end{aligned}
$$

Then the deuteron structure function can be related to the proton and neutron ones as it follows:

$$
\begin{aligned}
\mathcal{M}\left[x g_{1}^{d}, \mathrm{~N}\right]\left(Q^{2}\right)= & \frac{1}{2}\left(\mathcal{M}\left[x g_{1}^{p}, \mathrm{~N}\right]+\mathcal{M}\left[x g_{1}^{n}, \mathrm{~N}\right]\right) \\
& \times\left(1-\frac{3}{2} \omega_{D}\right) .
\end{aligned}
$$

In this equation $\omega_{D}=0.05 \pm 0.01$ denotes the $D$-state wave probability for the deuteron [69-73].

Based on Eq. (4) it is possible to present the $x g_{1}^{p}$, for instance at the initial scale $Q_{0}^{2}=1 \mathrm{GeV}^{2}$, in terms of 9 unknown parameters. These unknown parameters can now be extracted, using the global fit over the available data for the spin structure functions. Details of the fit will be illustrated in the following subsections.

\subsection{Jacobi polynomials technique}

Here we describe briefly the technique of Jacobi polynomials as a tool to do a global fit for the polarized structure functions over the experimental data. The advantage of this technique can be found in what have been done before $[65,66,74-79]$ and also in other studies such as [56,80-91]. According to this method the polarized structure functions $x g_{1}\left(x, Q^{2}\right)$ can be expanded in terms of the Jacobi polynomials $\Theta_{n}^{\alpha, \beta}(x)$ such that [87]

$x g_{1}\left(x, Q^{2}\right)=x^{\beta}(1-x)^{\alpha} \sum_{n=0}^{N_{\max }} a_{n}\left(Q^{2}\right) \Theta_{n}^{\alpha, \beta}(x)$.

Here the maximum order of expansion is denoting by $\mathrm{N}_{\max }$. Two free parameters $\alpha$ and $\beta$ are chosen to make the fastest convergence for the series in Eq. (9). The $a_{n}\left(Q^{2}\right)$ as Jacobi moment discloses the dependent of polarized structure functions on $\mathrm{Q}^{2}$. The weight function $w^{\alpha, \beta}(x) \equiv x^{\beta}(1-x)^{\alpha}$ performs the $x$-dependence of structure function that is accom- 
plished by Jacobi polynomials $\Theta_{n}^{\alpha, \beta}(x)$ through the following expansion:

$\Theta_{n}^{\alpha, \beta}(x)=\sum_{j=0}^{n} c_{j}^{(n)}(\alpha, \beta) x^{j}$.

It can be shown that the expansion coefficient $c_{j}^{(n)}(\alpha, \beta)$ is given by a combining of Gamma function in terms of $n$ order and $\alpha, \beta$ parameters.

The following orthogonality relation is satisfied by Jacobi polynomials:

$\int_{0}^{1} d x x^{\beta}(1-x)^{\alpha} \Theta_{n}^{\alpha, \beta}(x) \Theta_{l}^{\alpha, \beta}(x)=\delta_{n, l}$.

Based on the orthogonality condition the Jacobi moments, $a_{n}\left(Q^{2}\right)$, can be obtained as it follows [87]:

$$
\begin{aligned}
a_{n}\left(Q^{2}\right) & =\int_{0}^{1} d x x g_{1}\left(x, Q^{2}\right) \Theta_{n}^{\alpha, \beta}(x) \\
& =\sum_{j=0}^{n} c_{j}^{(n)}(\alpha, \beta) \mathcal{M}\left[x g_{1}, j+2\right]\left(Q^{2}\right),
\end{aligned}
$$

where the $\mathcal{M}\left[x g_{1}, j+2\right]\left(Q^{2}\right)$ is the Mellin transform of $x g_{1}\left(x, Q^{2}\right)$, defined by Eq. (3) [87].

Now by substituting Eq. (9) into Eq. (12) the polarized structure function $x g_{1}\left(x, Q^{2}\right)$, based on Jacobi polynomial expansion method, can be constructed. Therefore the following expression for $x g_{1}\left(x, Q^{2}\right)$ would be obtained [87]:

$$
\begin{aligned}
x g_{1}\left(x, Q^{2}\right)= & x^{\beta}(1-x)^{\alpha} \sum_{n=0}^{\mathrm{N}_{\max }} \Theta_{n}^{\alpha, \beta}(x) \\
& \times \sum_{j=0}^{n} c_{j}^{(n)}(\alpha, \beta) \mathcal{M}\left[x g_{1}, j+2\right]\left(Q^{2}\right) .
\end{aligned}
$$

Considering the whole kinematic region for polarized DIS data, the optimal convergence of expansion in Eq. (13) is achieved by setting $N_{\max }=9$ and $\alpha=3, \beta=0.5$. More details how to fix maximum order of expansion and the required Jacobi parameters can be found in[66].

\section{QCD fit of $\operatorname{xg}_{1}\left(x, Q^{2}\right)$ data}

We do our analysis, based on the QCD-PEGASUS program [92]. The QCD evolution is performed at the NLO accuracy with the massless partonic flavors $\{u, d, s\}$, using fixedflavor number scheme. It is assumed that the renormalization and factorization scales to be identical $\left(\mu_{R}=\mu_{F}\right)$ and the NLO coupling constant is taken into account by fourth order Runge-Kutta integration [93]. In order to make the fitting pro- cedure numerically efficient, the PPDFs parameterizations of Eq. (1) are followed in Mellin moment N-space.

The data sets which are used in the fit, includes E143 [94], HERMES [95,96], EMC [97], E155 [98], SMC [99], COMPASS [100-102] and Jlab [103,104] for the proton and E142 [105], HERMES [95,106], E154 [107] and Jlab [108-110] for the neutron. Finally we use SMC [99], E143 [94], E155 [111], HERMES [96], COMPASS [112-114] data sets for the deuteron. They are listed in Table 1. We are the first group who employ the recent data of Jlab [104] in the relevant QCD analyses. The data sets in Table 1 are related to the scattering cross sections of plorized beam from longitudinal polarized targets. These cross sections are related to the measured double spin asymmetries from which the spin structure functions $g_{1}^{p}$ and $g_{1}^{n}$ could be extracted. Details of analysis for a full data set can be found for instance in [102,104].

The fit is established by minimizing the global $\chi^{2}[65,66$, 75]:

$\chi_{\text {global }}^{2}=\sum_{n} w_{n} \chi_{n}^{2}$

In this equation the sum involves different experiments and the relevant weight factor for $n^{\text {th }}$ experiment is denoted by $w_{n}[115,116]$, which we take to be unity. This factor, with default value 1 , is obtained by physics considerations or other information as a prior knowledge. Consequently in any data analysis a priori choices for the $w_{n}$ are explicitly or implicitly present. In this regard data inclusion or omission will lead to extreme cases, assigning to each available experimental data set either $100 \%$ or $0 \%$ weight. These choices are varying for different global analysis efforts. In a similar manner the choices which are made for various elements of the analysis procedure are indicating subjective inputs.

The mathematical expression for $\chi_{n}^{2}$ has the following form

$\chi_{n}^{2}=\left(\frac{1-\mathcal{N}_{n}}{\Delta \mathcal{N}_{n}}\right)^{2}+\sum_{i}\left(\frac{\mathcal{N}_{n} g_{1, i}^{\text {exp }}-g_{1, i}^{\text {theor }}}{\mathcal{N}_{n} \Delta g_{1, i}^{\text {exp }}}\right)^{2}$.

For the $i^{\text {th }}$ data point, the experimental measurement, experimental uncertainty and theoretical value are presented by $g_{1, i}^{\text {exp }}, \Delta g_{1, i}^{\text {exp }}$, and $g_{1, i}^{\text {theor }}$ respectively. The rest of quantities are $\mathcal{N}_{n}$ as an overall normalization factor and $\Delta \mathcal{N}_{n}$ as the experimental normalization uncertainty for the data of $n$th experiment. We should note that the normalization factor $\mathcal{N}_{n}$ is allowed to be shifted among different data sets, considering the uncertainties $\Delta \mathcal{N}_{n}$ raised out from various experiments.

Using the CERN program library MINUIT $[48,49]$ the $\chi_{\text {global }}^{2}$ can be minimized and consequently the 9 unknown parameters, involving $\alpha_{s}\left(Q_{0}^{2}\right)$, are determined which are listed in Table 2. What is obtained for the numerical value 
Table 1 Summary of published polarized DIS experimental data points with measured $x$ and $Q^{2}$ ranges and the number of data points

\begin{tabular}{|c|c|c|c|c|c|c|}
\hline Experiment & Ref. & {$\left[x_{\min }, x_{\max }\right]$} & $\mathrm{Q}^{2}\left(\mathrm{GeV}^{2}\right)$ & Num. of data poi. & $\chi^{2}$ & $\mathcal{N}_{i}$ \\
\hline SLAC/E143(p) & [94] & [0.031-0.749] & $1.27-9.52$ & 28 & 23.2809 & 0.9982 \\
\hline HERMES(p) & [95] & {$[0.028-0.66]$} & $1.01-7.36$ & 39 & 47.3685 & 1.0004 \\
\hline $\mathrm{SMC}(\mathrm{p})$ & [99] & {$[0.005-0.480]$} & $1.30-58.0$ & 12 & 3.5157 & 0.9998 \\
\hline $\mathrm{EMC}(\mathrm{p})$ & [97] & {$[0.015-0.466]$} & $3.50-29.5$ & 10 & 5.6992 & 1.0055 \\
\hline SLAC/E155 & [98] & {$[0.015-0.750]$} & $1.22-34.72$ & 24 & 21.9195 & 1.0163 \\
\hline HERMES06(p) & [96] & {$[0.026-0.731]$} & $1.12-14.29$ & 51 & 22.3716 & 0.9975 \\
\hline COMPASS10(p) & {$[100,101]$} & {$[0.005-0.568]$} & $1.10-62.10$ & 15 & 17.2058 & 0.9903 \\
\hline COMPASS16(p) & [102] & {$[0.0035-0.575]$} & $1.03-96.1$ & 54 & 34.0399 & 0.9990 \\
\hline SLAC/E143(p) & [94] & [0.031-0.749] & $2-3-5$ & 84 & 95.6372 & 0.9983 \\
\hline HERMES(p) & [95] & {$[0.023-0.66]$} & 2.5 & 20 & 29.0133 & 1.0013 \\
\hline $\mathrm{SMC}(\mathrm{p})$ & [99] & {$[0.003-0.4]$} & 10 & 12 & 11.6274 & 1.0007 \\
\hline Jlab06(p) & {$[103]$} & [0.3771-0.9086] & $3.48-4.96$ & 70 & 101.6514 & 1.0003 \\
\hline Jlab17(p) & [104] & [0.37696-0.94585] & $3.01503-5.75676$ & 82 & 192.7984 & 1.0017 \\
\hline$g_{1}^{p}$ & & & & 501 & & \\
\hline SLAC/E143(d) & [94] & [0.031-0.749] & $1.27-9.52$ & 28 & 38.8430 & 0.9991 \\
\hline SLAC/E155(d) & [111] & {$[0.015-0.750]$} & $1.22-34.79$ & 24 & 18.0728 & 0.9999 \\
\hline $\mathrm{SMC}(\mathrm{d})$ & [99] & {$[0.005-0.479]$} & $1.30-54.80$ & 12 & 16.8776 & 0.9999 \\
\hline HERMES06(d) & [96] & {$[0.026-0.731]$} & $1.12-14.29$ & 51 & 46.5585 & 0.9979 \\
\hline COMPASS05(d) & [112] & [0.0051-0.4740] & $1.18-47.5$ & 11 & 8.5519 & 0.9978 \\
\hline COMPASS06(d) & [113] & {$[0.0046-0.566]$} & $1.10-55.3$ & 15 & 8.5539 & 0.9999 \\
\hline COMPASS17(d) & [114] & {$[0.0045-0.569]$} & $1.03-74.1$ & 43 & 28.0897 & 1.0008 \\
\hline SLAC/E143(d) & [94] & [0.031-0.749] & $2-3-5$ & 84 & 127.1429 & 0.9995 \\
\hline$g_{1}^{d}$ & & & & 268 & & \\
\hline SLAC/E142(n) & [105] & {$[0.035-0.466]$} & $1.10-5.50$ & 8 & 6.9193 & 0.9989 \\
\hline HERMES(n) & [95] & {$[0.033-0.464]$} & $1.22-5.25$ & 9 & 2.5946 & 0.9999 \\
\hline E154(n) & [107] & {$[0.017-0.564]$} & $1.20-15.00$ & 17 & 16.1326 & 0.9994 \\
\hline HERMES06(n) & [106] & {$[0.026-0.731]$} & $1.12-14.29$ & 51 & 18.0867 & 0.9999 \\
\hline Jlab03(n) & [108] & [0.14-0.22] & $1.09-1.46$ & 4 & $3.817 \mathrm{e}-2$ & 0.9998 \\
\hline Jlab04(n) & [109] & [0.33-0.60] & $2.71-4.8$ & 3 & 0.7391 & 0.9999 \\
\hline Jlab05(n) & [110] & [0.19-0.20] & $1.13-1.34$ & 2 & 1.0738 & 1.0103 \\
\hline$g_{1}^{n}$ & & & & 94 & & \\
\hline Total & & & & 863 & 944.4047 & \\
\hline
\end{tabular}

Table 2 Numerical values for the concerned parameters and their statistical errors at the input scale $Q_{0}^{2}=1 \mathrm{GeV}^{2}$

\begin{tabular}{|c|c|c|c|c|c|}
\hline \multirow[t]{4}{*}{$\delta u_{v}$} & $\eta_{u_{v}}$ & 0.928 (fixed) & $\delta \bar{q}$ & $\eta_{\bar{q}}$ & $-0.0533 \pm 0.0106$ \\
\hline & $\alpha_{u_{v}}$ & $0.3207 \pm 0.015$ & & $\alpha_{\bar{q}}$ & $0.5342 \pm 0.468$ \\
\hline & $\beta_{u_{v}}$ & $2.626 \pm 0.033$ & & $\beta_{\bar{q}}$ & 8.024 (fixed) \\
\hline & $\gamma_{u_{v}}$ & 28.505 (fixed) & & $\gamma_{\bar{q}}$ & 0 \\
\hline \multirow[t]{4}{*}{$\delta d_{v}$} & $\eta_{d_{v}}$ & $-0.342($ fixed $)$ & $\delta g$ & $\eta_{g}$ & $0.1777 \pm 0.0895$ \\
\hline & $\alpha_{d_{v}}$ & $0.1184 \pm 0.017$ & & $\alpha_{g}$ & $6.9804 \pm 1.948$ \\
\hline & $\beta_{d_{v}}$ & $3.536 \pm 0.207$ & & $\beta_{g}$ & $4.953($ fixed $)$ \\
\hline & $\gamma_{d_{v}}$ & 97.831 (fixed) & & $\gamma_{g}$ & 0 \\
\hline \multicolumn{6}{|c|}{$\alpha_{s}\left(Q_{0}^{2}\right)=0.5408 \pm 0.0428$} \\
\hline \multicolumn{6}{|c|}{$\chi^{2} /$ dof $=944.4047 / 854=1.10586$} \\
\hline
\end{tabular}


of coupling constant at energy scale $Q_{0}$ with $0.68 \%$ confidence level is $\alpha_{s}\left(Q_{0}^{2}\right)=0.5408 \pm 0.0428$. By changing the energy scale to the $\mathrm{Z}$ boson mass, we achieve $\alpha_{s}\left(M_{Z}^{2}\right)=$ $0.1143 \pm 0.00005$, which is consistent with the value obtained in Ref.[117] $\left(\alpha_{s}\left(M_{Z}^{2}\right)=0.1146 \pm 0.0021\right)$, while the present world average value is $\alpha_{s}\left(M_{Z}^{2}\right)=0.1179 \pm 8.5 \times 10^{-6}$ [118]. In this regard an acceptable fit to the experimental data is obtained with $\chi^{2}$ d.o.f. $=1.1059$.

\subsection{Polarized PDFs comparison}

The result of PPDFs as a function of $x$ at $\mathrm{Q}_{0}^{2}=1 \mathrm{GeV}^{2}$ along with the corresponding uncertainty bounds are depicted in Fig. 1. For comparison various parametrization models like BB [61], GRSV [119], LSS05 [120] and KATAO [74] at the NLO accuracy have also been added there.

The uncertainty estimation is computed, using the Hessian method [50]. The best determined distributions, raised out from the inclusive polarized DIS data are the $x \Delta u_{v}$ and $x \Delta d_{v}$ densities while the preceding one has smaller uncertainty band with respect to the $x \Delta d_{v}$ density. As can be seen our results for polarized valence densities are compatible with parametrization models while the ones for $x \Delta \bar{q}$ and $x \Delta g$ densities behave differently with respect to the models. In the $x<10^{-3}$ and $x>0.8$ regions the valence polarized PDFs are not constrained by data, obtained by extrapolation and are corresponding with the models.

\subsection{Polarized structure function comparison}

To predict the properties of PPDFs at small and large- $x$ values, it is needed to study the nucleon structure functions extensively. The accuracy of achieved PPDFs can be checked to accomplish DIS data by comparing the extracted structure functions with the available data.

The analyses of different parametrization groups can be separated by taking into account the more numerous and accurate data at both small and large- $x$ regions. Our predictions from Jocobi polynomials technique and the results from different models for polarized structure functions of the proton $x g_{1}^{p}\left(x, Q^{2}\right)$, neutron $g_{1}^{n}\left(x, Q^{2}\right)$ and deuteron $x g_{1}^{d}\left(x, Q^{2}\right)$ are presented in Figs. 2, 3, 4, 5 and 6. Comparison with the fixed-target DIS experimental data from E143 [94], Jlab06 [103], Jlab17 [104], E154 [107], Jlab03 [108], Jlab04 [109], Jlab05 [110] and COMPASS [100-102,112-114] are also done there. The models which are added in these figures are BB [61], GRSV [119], LSS05 [120] and KATAO [74]. Our results which are presented for some selected values of $Q^{2}=$ $2,3.48,4.4,1.5,5,2$ and $13 \mathrm{GeV}^{2}$ are in good agreement with the experimental data and in accordance with other determinations over the entire range of $x$ and $\mathrm{Q}^{2}$.
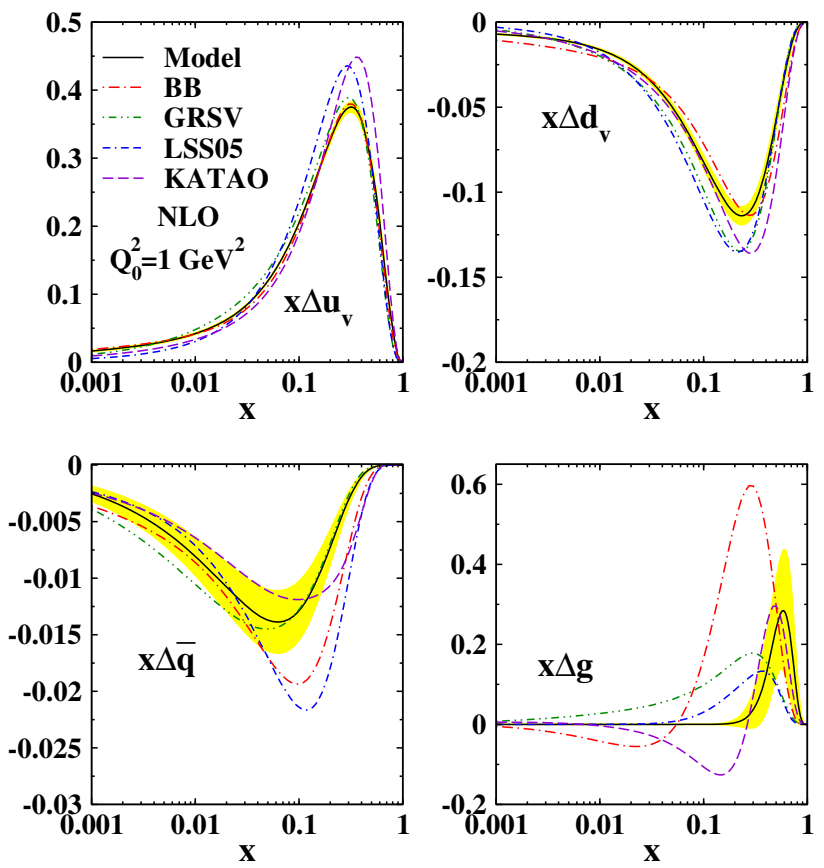

Fig. 1 The polarized parton distribution at $Q_{0}^{2}=1 \mathrm{GeV}^{2}$ as a function of $x$. Our fit is the solid curve. The results of parametrization models like BB (dashed-dotted) [61], GRSV (dashed-dotted-dotted) [119], LSS05 (dashed-dashed-dotted) [120] and KATAO (dashed) [74] have also been shown

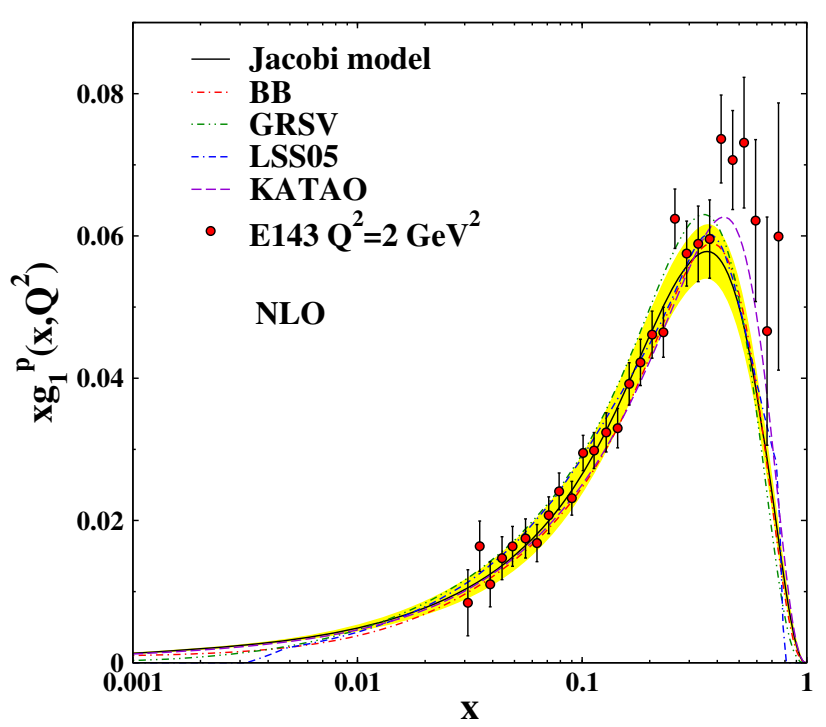

Fig. 2 The polarized proton structure functions as a function of $x$ at $Q^{2}=2 \mathrm{GeV}^{2}$. The result of Jacobi expansion technique (solid curve) is compared with parametrization models like BB (dashed-dotted) [61], GRSV (dashed-dotted-dotted) [119], LSS05 (dashed-dashed-dotted) [120] and KATAO (dashed) [74]

\section{Transverse momentum dependence of polarized PDFs}

In this section we investigate the transverse momentum dependence of the spin-dependent quark distribution func- 


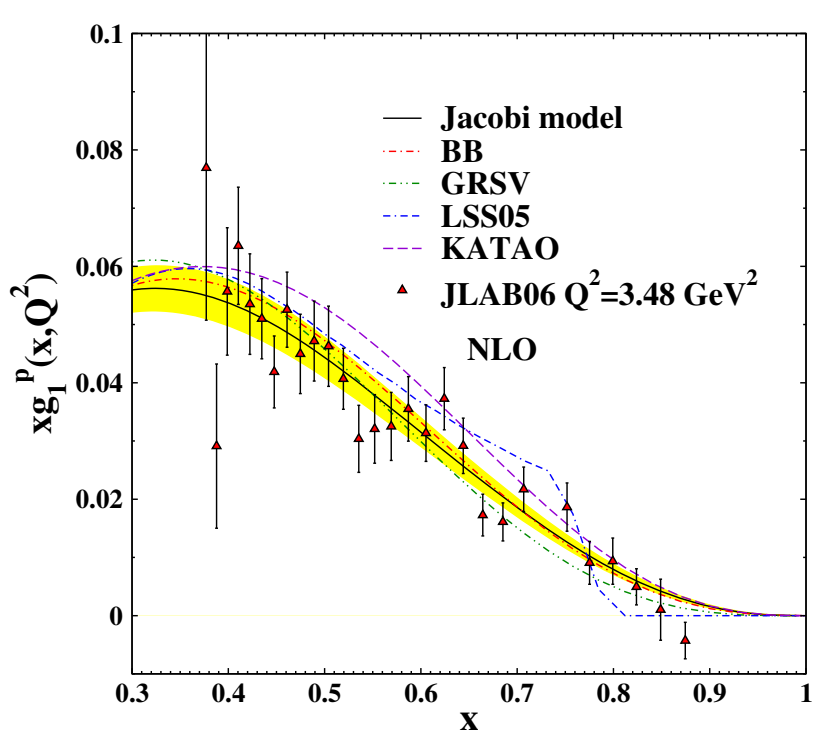

Fig. 3 As in Fig.2 but at $Q^{2}=3.5 \mathrm{GeV}^{2}$ and in a shorter range of $x$ which is corresponding to the available data

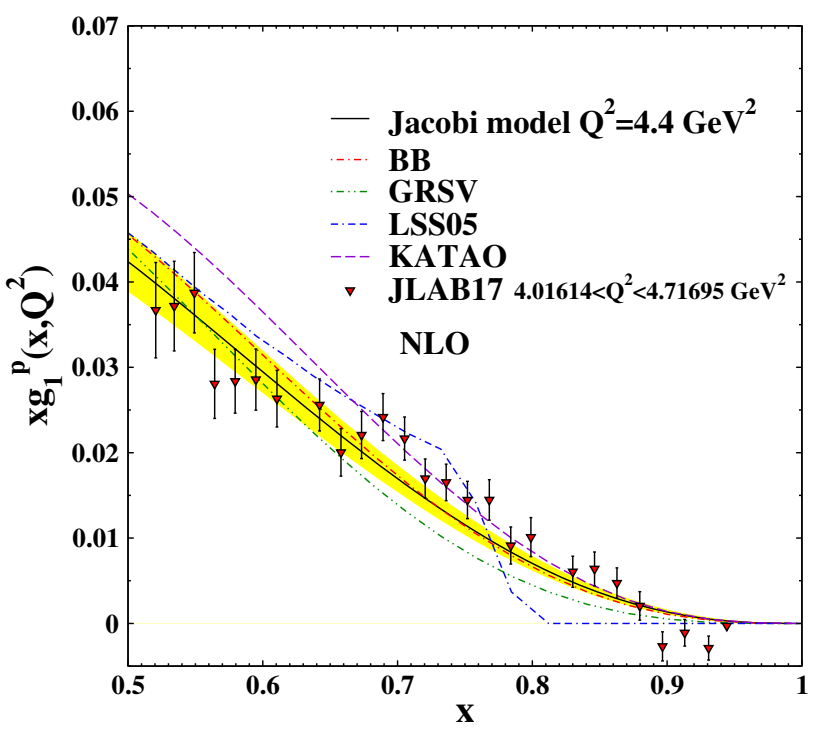

Fig. 4 As in Fig.2 but at $Q^{2}=4.4 \mathrm{GeV}^{2}$ and in a shorter range of $x$ which is corresponding the available data

tions applying the theoretical approach of Ref. [47]. In this approach, the dependence of polarized quark distributions on transverse momentum of quark, $k_{T}$, is obtained by imposing Lorentz covariance in standard parton model and these transverse momentum dependent polarized quark distributions $\left(\Delta q\left(x, k_{T}\right)\right)$ are related to the integrated ones $(\Delta q(x))$ [47]. Correspondingly partons can have transverse momentum with respect to direction of nucleon as parent hadron.

The spin-dependent structure function, $g_{1}$ is written in terms of polarized parton distribution functions in standard collinear parton model $[39,41,45,47]$. In the framework of Ref. [47], the integrated polarized quark distribution function
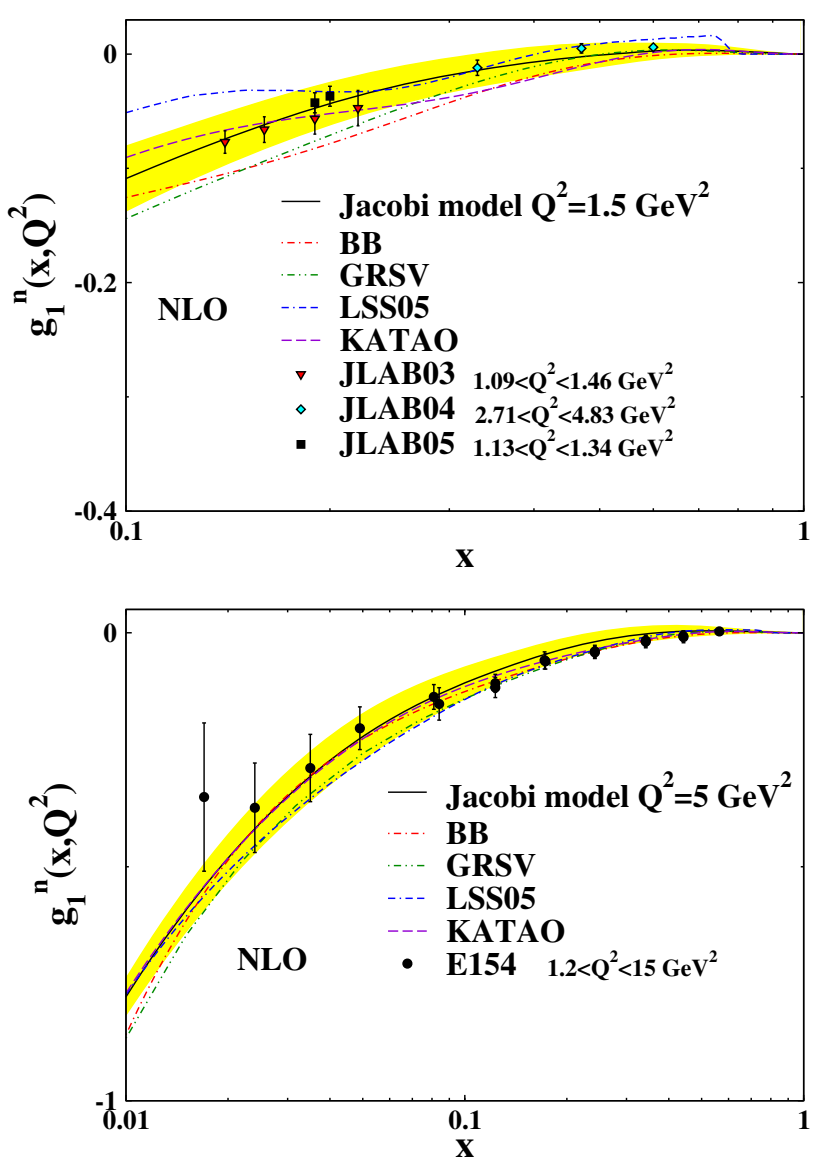

Fig. 5 The spin dependent neutron structure functions as a function of $x$ at $Q^{2}=1.5 \mathrm{GeV}^{2}$ and $5 \mathrm{GeV}^{2}$. The result of Jacobi expansion technique (solid curve) is compared with parametrization models like BB (dashed-dotted) [61], GRSV (dashed-dotted-dotted) [119], LSS05 (dashed-dashed-dotted) [120] and KATAO (dashed) [74]

is defined as:

$\Delta q(x)=x \int_{x}^{1} d \eta(\eta-2 x) \varphi_{8}(\eta)$

By considering the following relation [47]:

$\int_{\xi}^{1} \frac{d \eta}{\eta} \Delta q(\eta)=\int_{\xi}^{1} d \eta \int_{\eta}^{1} d \eta^{\prime}\left(\eta^{\prime}-2 \eta\right) \varphi_{8}\left(\eta^{\prime}\right)$,

which is written using Eq. (16), and changing the order of integral as:

$\int_{\xi}^{1} \frac{d \eta}{\eta} \Delta q(\eta)=\xi \int_{\xi}^{1} d \eta^{\prime}\left(\xi-\eta^{\prime}\right) \varphi_{8}\left(\eta^{\prime}\right)$,

the relation between the distributions $\Delta q$ and $\varphi_{8}$ is modified as [47]:

$\xi \int_{\xi}^{1} d \eta \varphi_{8}(\eta)=-\frac{1}{\xi}\left[\Delta q(\xi)+\int_{\xi}^{1} \frac{d \eta}{\eta} \Delta q(\eta)\right]$. 

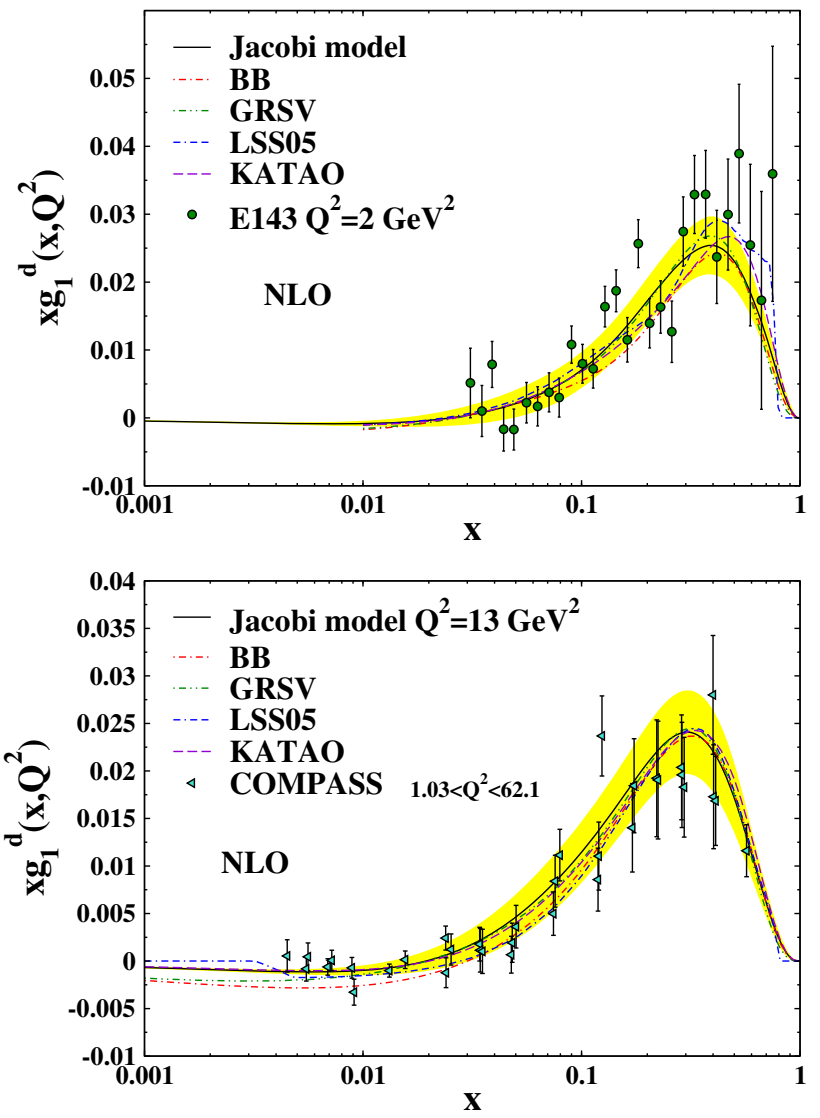

Fig. 6 The spin dependent of deutron structure functions as a function of $x$ at $Q^{2}=2 \mathrm{GeV}^{2}$ and $13 \mathrm{GeV}^{2}$. The result of Jacobi expansion technique (solid curve) is compared with parametrization models like BB (dashed-dotted) [61], GRSV (dashed-dotted-dotted) [119], LSS05 (dashed-dashed-dotted) [120] and KATAO (dashed) [74]

Hence, $\varphi_{8}(\eta)$ can be written as $[39,41,45,47]$ :

$\varphi_{8}(\eta)=\frac{-1}{\eta^{3}}\left[3 \Delta q(\eta)-\eta \frac{d}{d \eta}(\Delta q(\eta))+2 \int_{\eta}^{1} \frac{d \eta^{\prime}}{\eta^{\prime}} \Delta q\left(\eta^{\prime}\right)\right]$,

which shows that $\varphi_{8}$ distribution is computable from the functional form of integrated polarized quark distribution function.

Finally by considering Eq. (16) and the definition of $\eta$ parameter [47], $\eta=\left(x+\frac{k_{T}^{2}}{x M^{2}}\right)$ ( $M$ is the mass of nucleon $)$,

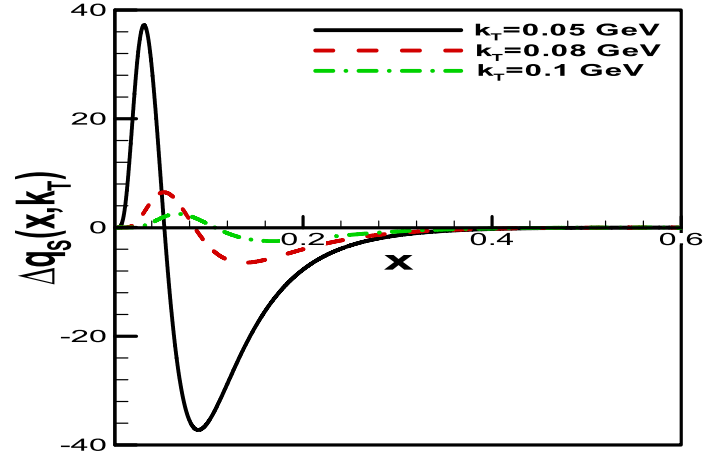

Fig. 8 The $x$-distribution of total TMD polarized sea quarks at $Q_{0}^{2}=$ $1 \mathrm{GeV}^{2}$

the TMD polarized quark distribution is obtained as [47]:

$$
\begin{aligned}
\Delta q\left(x, k_{T}\right)= & -\frac{1}{\pi M^{2}} x\left[1-\frac{k_{T}^{2}}{x^{2} M^{2}}\right] \varphi_{8}\left(x\left(1+\frac{k_{T}^{2}}{x^{2} M^{2}}\right)\right) \\
& \times \theta\left(x(1-x) M^{2}-k_{T}^{2}\right) .
\end{aligned}
$$

Considering Eqs. (20) and (21), the dependence of polarized quark distribution on $k_{T}$ can be obtained from the $x-$ dependence of corresponding integrated quark distribution. Therefore, to calculate the TMD polarized quark distributions, it is first needed to determine the integrated spindependent quark distributions of proton. We have obtained these integrated polarized distributions using a global analysis of recent experimental data in Sects. 2 and 3.

\section{Results of TMD polarized densities}

We calculate the TMD polarized quark distribution functions of proton. For this purpose, we apply a theoretical model in which the transverse momentum dependence of the quark distributions are obtained by considering Lorentz structure in the parton model [47]. In order to compute the TMD polarized distributions, using the mentioned theoretical approach, we should first obtain the integrated spin-dependent quark distribution functions. To this end, we calculate the PPDFs of proton using a global analysis of recent experimental data
Fig. 7 The TMD polarized a up and $\mathbf{b}$ down valence quark distribution of proton with respect to $x$ at three $k_{T}$ values and $Q_{0}^{2}=1 \mathrm{GeV}^{2}$
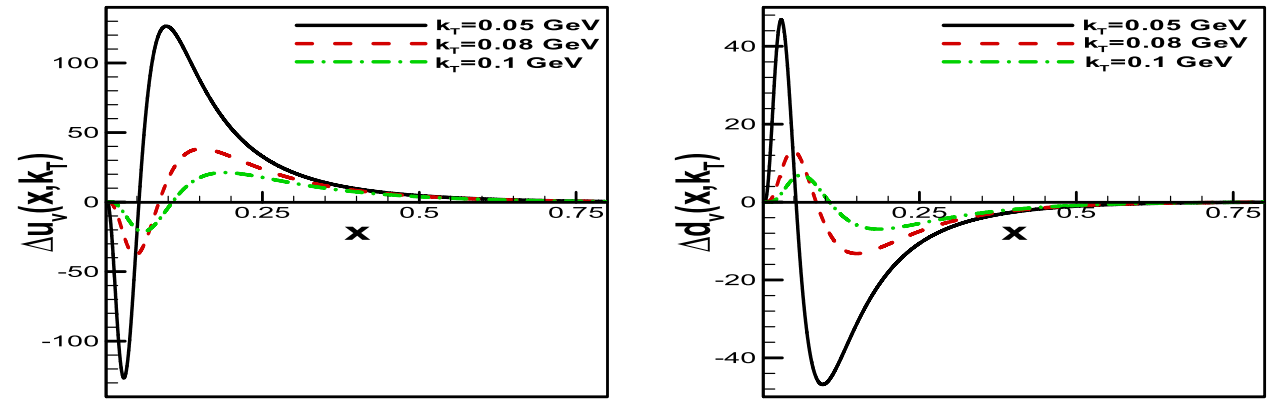

(a) 
Fig. 9 The TMD polarized a up and $\mathbf{b}$ down valence quark distribution of proton with respect to $k_{T}$ at three values of $x$ and $Q_{0}^{2}=1 \mathrm{GeV}^{2}$

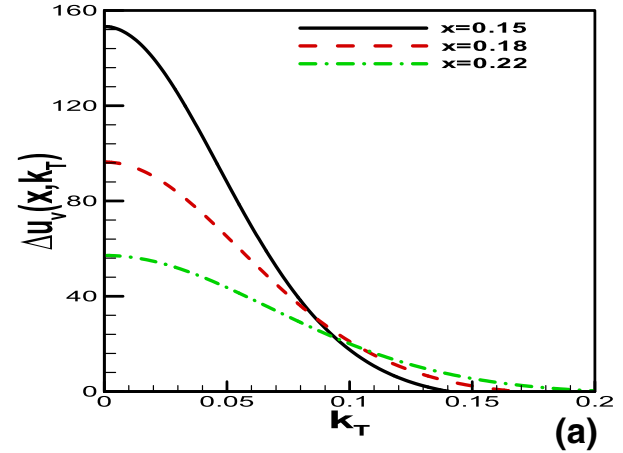

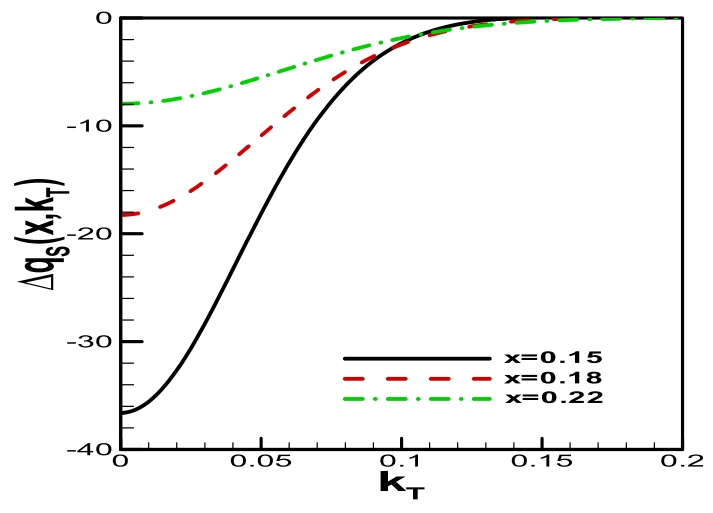

Fig. 10 The $k_{T}$-distribution of total TMD polarized sea quarks at $Q_{0}^{2}=$ $1 \mathrm{GeV}^{2}$

at $Q_{0}^{2}=1 \mathrm{Ge} \mathrm{V}^{2}$ which has been explained extensively in Sects. 2 and 3.

In Fig. 7, we present the TMD polarized valence quark distributions, $\Delta u_{v}\left(x, k_{T}\right)$ and $\Delta d_{v}\left(x, k_{T}\right)$, with respect to $x$ at fixed values of transverse momentum, $k_{T}=0.05 \mathrm{GeV}$, $0.08 \mathrm{GeV}$ and $0.1 \mathrm{GeV}$, at $Q_{0}^{2}=1 \mathrm{GeV}^{2}$. It is found that by increasing $k_{T}$ value, the probability for finding the polarized valence quarks decreases and the TMD polarized valence distributions have the shifts toward the larger values of $x$.

We display the total TMD polarized sea quark distribution of proton, $\Delta q_{S}\left(x, k_{T}\right)=6 \Delta \bar{q}\left(x, k_{T}\right)$, at $Q_{0}^{2}=1 \mathrm{GeV}^{2}$ in Fig. 8. This $x$-distribution is plotted at three $k_{T}$ values. It can be seen that increasing the value of transverse momentum affects the TMD polarized sea distribution more considerably in comparison with the valence one.

In Figs. 9 and 10, we depict the results of TMD polarized valence and sea quark distributions, respectively, with respect to transverse momentum at $x=0.15,0.18$ and 0.22 . It should be pointed that the width of these spin-dependent $k_{T}$-distributions, which are very close to Gaussian distributions [44], changes by increasing the value of $x$.

From Figs. 7, 8, 9, 10, it is found that the results of our calculations for TMD polarized quark distribution functions show appropriate general treatment which is expected for TMD distributions [44-47].
In further step of our calculations, we first evolve the integrated polarized quark distribution functions of proton to higher $Q^{2}$ values using DGLAP evolution equations [2-5] at NLO approximation and then investigate their transverse momentum dependence applying Eq. (21).

In Figs. 11, 12 and 13, we present the results of our calculations for $x$-distribution of polarized valence and sea quarks at $Q^{2}=2 \mathrm{GeV}^{2}, Q^{2}=4 \mathrm{GeV}^{2}$ and $Q^{2}=10 \mathrm{GeV}^{2}$. These $x$-distributions are plotted at three fixed values of transverse momentum. It is found from these figures that at each value of $k_{T}$, the width of polarized $x$-distribution is dependent on $Q^{2}$ value. This property appears more obviously in TMD polarized distributions of sea quarks as expected.

In Figs. 14, 15 and 16, we plot the spin-dependent valence and sea quark $k_{T}$-distributions of proton at fixed values of $x$. In these figures we present each $k_{T}$-distribution at three values of $Q^{2}$. It can be seen from these figures that by increasing $Q^{2}$ value the width of polarized distributions particularly sea distribution, at each value of $x$, decreases. Furthermore, the polarized sea quark $k_{T}$-distribution falls off down by decreasing the value of $Q^{2}$ at each $x$ value. In general, we can say that the variation of TMD polarized sea quark distribution with respect to $Q^{2}$ is more considerable in comparison with the valence ones as expected.

\section{Conclusions}

We have studied the transverse momentum dependent polarized structure of proton. We have applied a theoretical approach to calculate the TMD spin-dependent valence and sea quark distribution functions of proton. In this approach, the transverse momentum dependence is taken into account by imposing Lorentz structure in standard parton model [47] and the dependence of polarized quark distributions on transverse momentum is obtained via the $x$-dependence of corresponding integrated polarized distributions [47]. Hence, in order to calculate the TMD polarized quark distributions of proton, we have first determined the integrated ones. Using Jacobi polynomial technique a fit to the polarized leptonDIS data on nucleon have been presented at the NLO accuracy. We find good agreement with the experimental data 


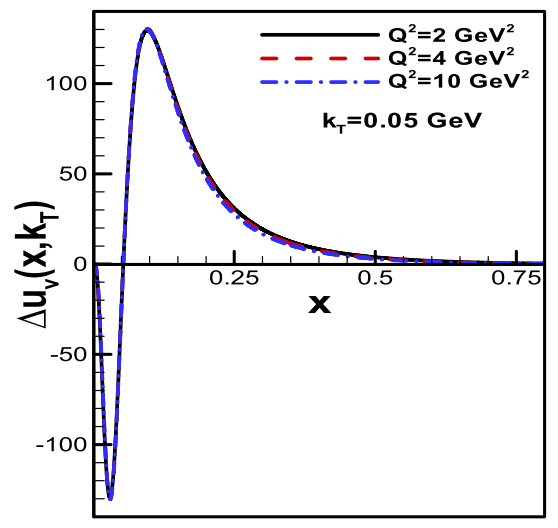

(a)

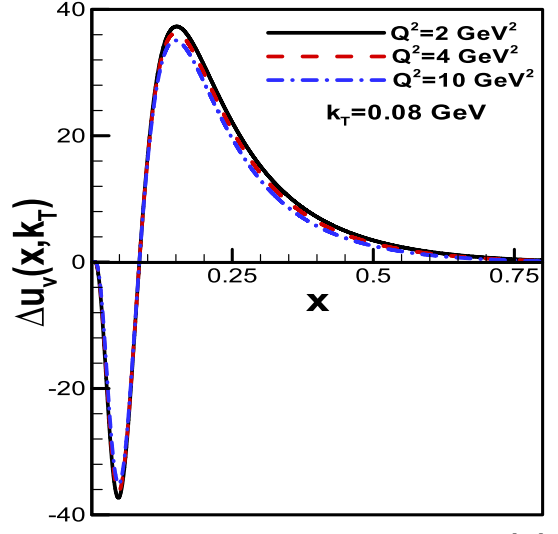

(b)

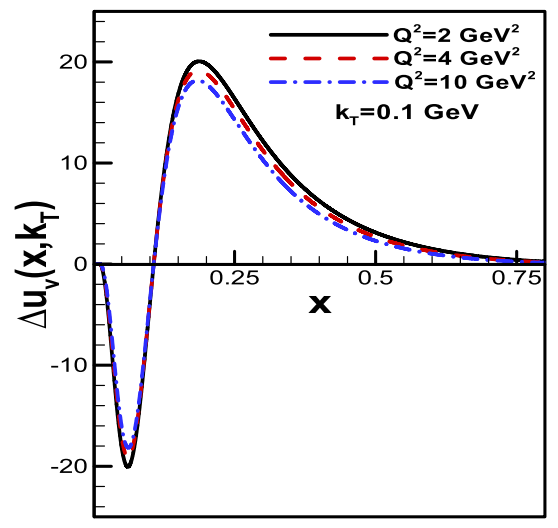

(c)

Fig. 11 The $x$-distribution of polarized up valence quark at $\mathbf{a} k_{T}=0.05 \mathrm{GeV}, \mathbf{b} k_{T}=0.08 \mathrm{GeV}$ and $\mathbf{c} k_{T}=0.1 \mathrm{GeV}$. The TMD polarized valence distributions are plotted at three values of $Q^{2}$

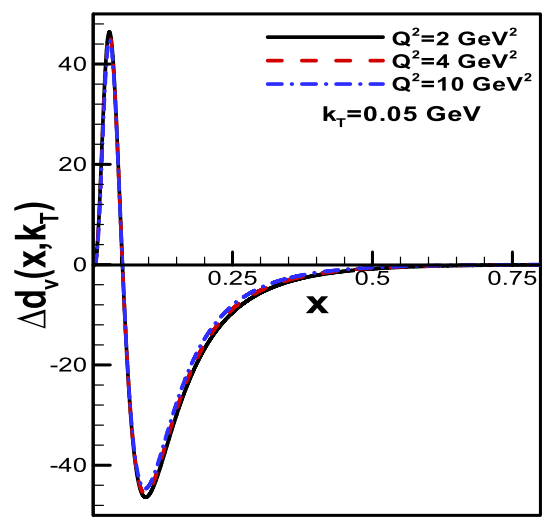

(a)

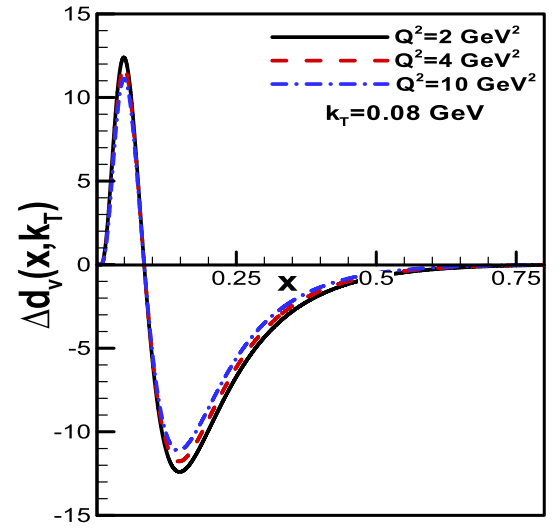

(b)

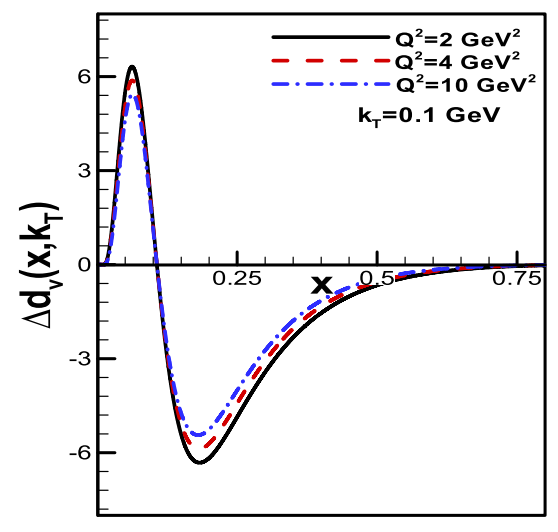

(c)

Fig. 12 The $x$-distribution of polarized down valence quark at a $k_{T}=0.05 \mathrm{GeV}, \mathbf{b} k_{T}=0.08 \mathrm{GeV}$ and $\mathbf{c} k_{T}=0.1 \mathrm{GeV}$. The TMD polarized valence distributions are plotted at three values of $Q^{2}$

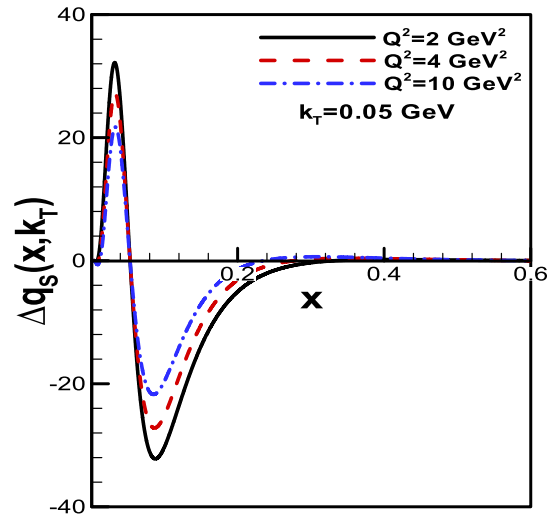

(a)

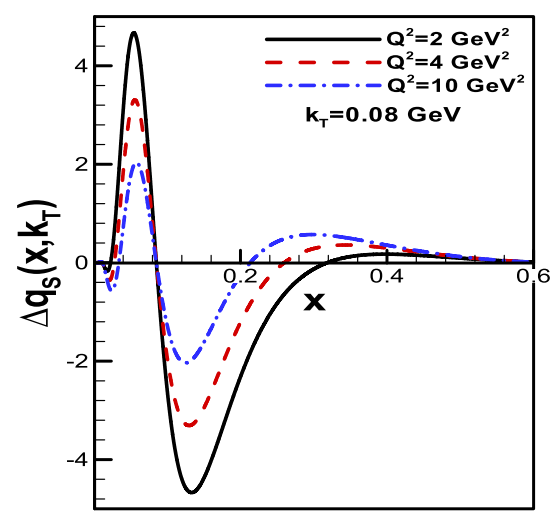

(b)

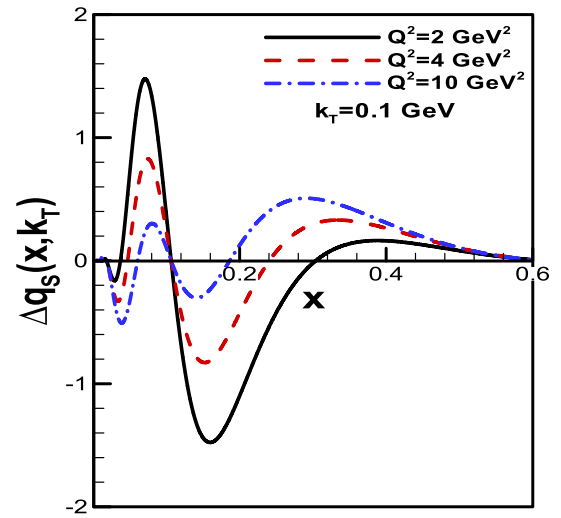

(c)

Fig. 13 The $x$-distribution of total polarized sea quarks at a $k_{T}=0.05 \mathrm{GeV}, \mathbf{b} k_{T}=0.08 \mathrm{GeV}$ and $\mathbf{c} k_{T}=0.1 \mathrm{GeV}$. The TMD polarized sea distributions are plotted at three values of $Q^{2}$ 

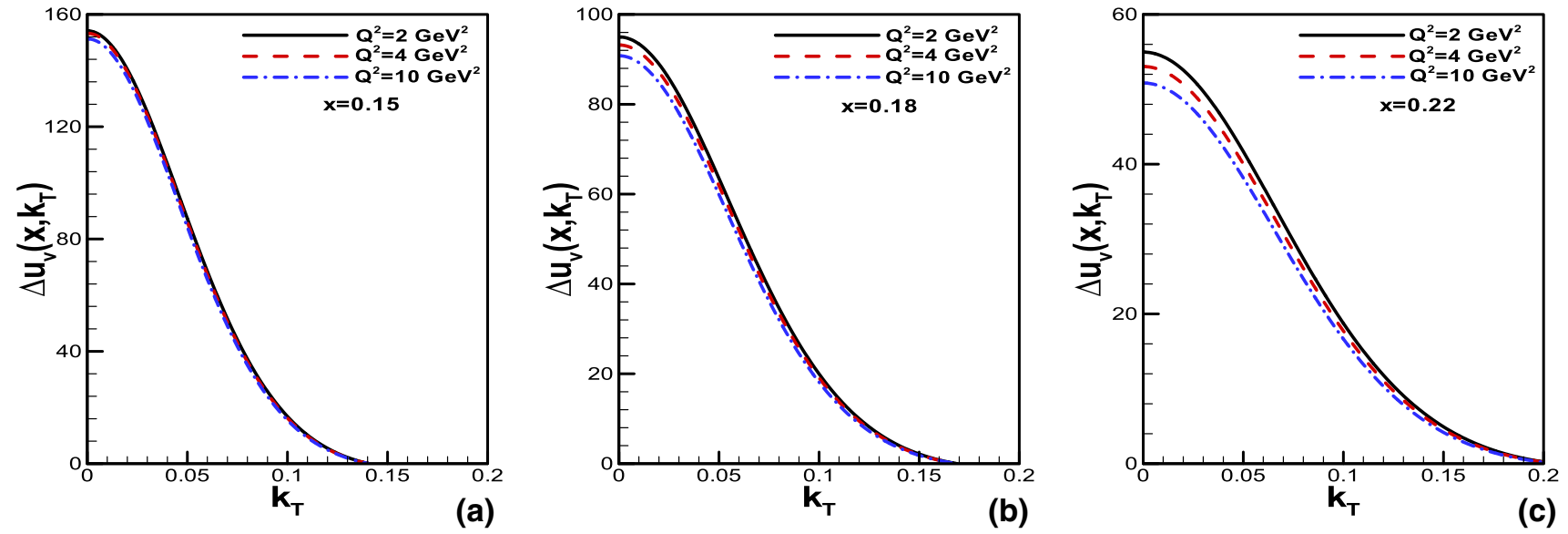

Fig. 14 The $k_{T}$-distribution of polarized up valence quark at $\mathbf{a} x=0.15, \mathbf{b} x=0.18$ and $\mathbf{c} x=0.22$. The TMD polarized valence distributions are plotted at three values of $Q^{2}$
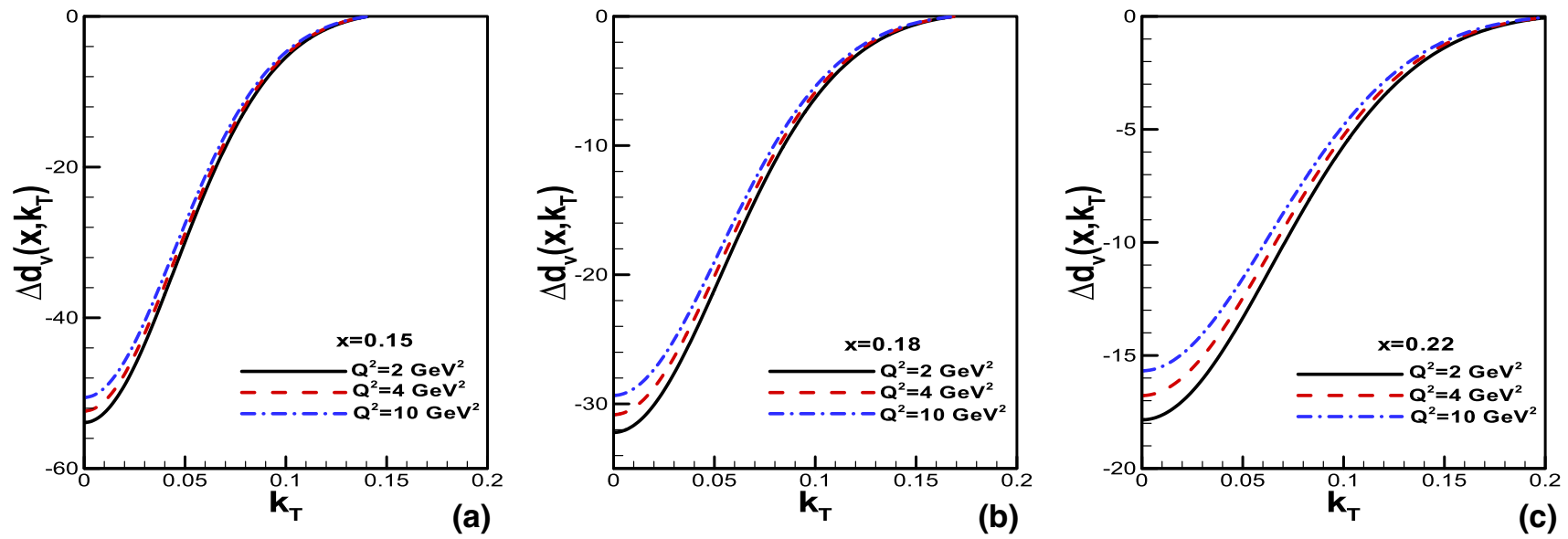

Fig. 15 The $k_{T}$-distribution of polarized down valence quark at $\mathbf{a} x=0.15, \mathbf{b} x=0.18$ and $\mathbf{c} x=0.22$. The TMD polarized valence distributions are plotted at three values of $Q^{2}$
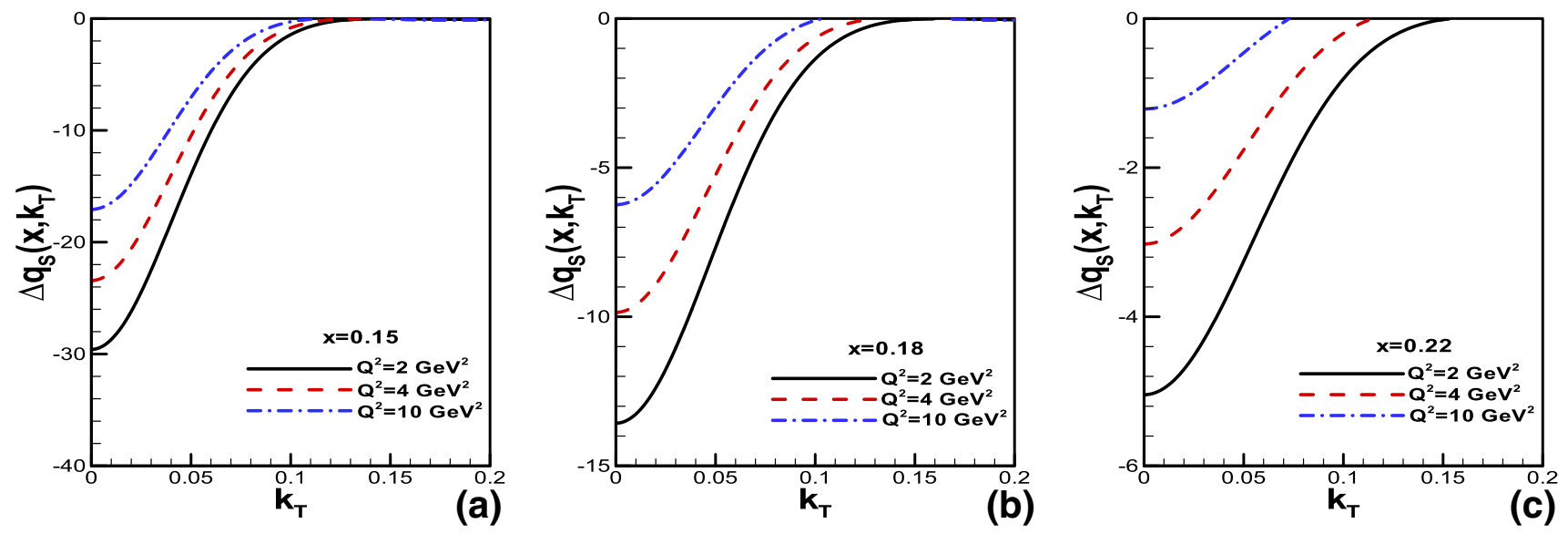

Fig. 16 The $k_{T}$-distribution of total polarized sea quarks at $\mathbf{a} x=0.15, \mathbf{b} x=0.18$ and $\mathbf{c} x=0.22$. The TMD polarized sea distributions are plotted at three values of $Q^{2}$ 
and our results are in correspond with determinations from parametrization models. In general we can demonstrate that an acceptable progressive has been achieved to describe the spin structure of the nucleon.

In further step, we have computed the TMD polarized valence and sea distributions of proton at $Q_{0}^{2}=1 \mathrm{GeV}^{2}$. The results of our calculations for these distributions show acceptable general behaviour at fixed values of $x$ and $k_{T}$. By evolving the initial integrated quark distributions to higher $Q^{2}$ scales, we have obtained the TMD polarized valence and sea quark distributions at $Q^{2}=2 \mathrm{GeV}^{2}, Q^{2}=4 \mathrm{GeV}^{2}$ and $Q^{2}=10 \mathrm{GeV}^{2}$. It is found that changing $Q^{2}$ value affects TMD polarized sea distribution more considerably in comparison with the valence ones as expected. As a further research task one can employ the polarized TMD to investigate transversity function. Following that considering the transverse spin one can investigate the single spin asymmetry in SIDIS processes. No one of these can be done without a complete knowledge of polarized TMDs which we hope to report on these issues as our future research activities.

Data Availability Statement This manuscript has no associated data or the data will not be deposited. [Authors' comment: The required data can be found in the text and references of this article.]

Open Access This article is licensed under a Creative Commons Attribution 4.0 International License, which permits use, sharing, adaptation, distribution and reproduction in any medium or format, as long as you give appropriate credit to the original author(s) and the source, provide a link to the Creative Commons licence, and indicate if changes were made. The images or other third party material in this article are included in the article's Creative Commons licence, unless indicated otherwise in a credit line to the material. If material is not included in the article's Creative Commons licence and your intended use is not permitted by statutory regulation or exceeds the permitted use, you will need to obtain permission directly from the copyright holder. To view a copy of this licence, visit http://creativecomm ons.org/licenses/by/4.0/.

Funded by SCOAP ${ }^{3}$.

\section{References}

1. Z.B. Kang et al., Phys. Rev. Lett 112, 102001 (2014)

2. Y.L. Dokshitzer, Sov. Phys. JETP 46, 641 (1977). [Zh. Eksp. Teor. Fiz. 73, 1216 (1977)]

3. V.N. Gribov, L.N. Lipatov, Sov. J. Nucl. Phys. 15, 438 (1972). [Yad. Fiz. 15, 781 (1972)]

4. L.N. Lipatov, Sov. J. Nucl. Phys. 20, 94 (1975). [Yad. Fiz. 20, 181 (1974)]

5. G. Altarelli, G. Parisi, Nucl. Phys. B 126, 298 (1977)

6. W. Bentz, I.C. Cloet, T. Ito, A.W. Thomas, K. Yazaki, Prog. Part. Nucl. Phys. 61, 238 (2008)

7. Y. Kahn, W. Melnitchouk, S.A. Kulagin, Phys. Rev. C 79, 035205 (2009)

8. M. Anselmino, M. Boglione, F. Murgia, Phys. Lett. B 362, 164 (1995)

9. M. Anselmino, M. Boglione, U. D’Alesio, A. Kotzinian, F. Murgia, A. Prokudin, C. Turk, Phys. Rev. D 75, 054032 (2007)

10. M. Anselmino, M. Boglione, U. D'Alesio, A. Kotzinian, F. Murgia, A. Prokudin, S. Melis, Nucl. Phys. Proc. Suppl. 191, 98 (2009)
11. M. Anselmino, M. Boglione, U. D’Alesio, S. Melis, F. Murgia, A. Prokudin, Phys. Rev. D 87, 094019 (2013)

12. Z.B. Kang, A. Prokudin, P. Sun, F. Yuan, Phys. Rev. D 91, 071501 (2015)

13. Z.B. Kang, A. Prokudin, P. Sun, F. Yuan, Phys. Rev. D 93(1), 014009 (2016)

14. H.W. Lin, W. Melnitchouk, A. Prokudin, N. Sato, H. Shows, Phys. Rev. Lett. 120(15), 152502 (2018)

15. S.J. Brodsky, D.S. Hwang, I. Schmidt, Phys. Lett. B 530, 99 (2002)

16. D.W. Sivers, Phys. Rev. D 41, 83 (1990)

17. A. Kotzinian, Nucl. Phys. B 441, 234 (1995)

18. J. Adams et al. [STAR Collaboration], Phys. Rev. Lett. 92, 171801 (2004)

19. S.S. Adler et al. [PHENIX Collaboration], Phys. Rev. Lett. 95, 202001 (2005)

20. B.I. Abelev et al. [STAR Collaboration], Phys. Rev. Lett. 99, 142003 (2007)

21. B.I. Abelev et al. [STAR Collaboration], Phys. Rev. Lett. 101, 222001 (2008)

22. I. Arsene et al. [BRAHMS Collaboration], Phys. Rev. Lett. 101, 042001 (2008)

23. L. Adamczyk et al. [STAR Collaboration], Phys. Rev. D 86, 051101 (2012)

24. E.C. Aschenauer et al., arXiv:1501.01220 [nucl-ex]

25. A. Airapetian et al. [HERMES Collaboration], Phys. Rev. Lett. 84, 4047 (2000)

26. A. Airapetian et al. [HERMES Collaboration], Phys. Rev. Lett. 94, 012002 (2005)

27. A. Airapetian et al. [HERMES Collaboration], Phys. Rev. Lett. 103, 152002 (2009)

28. A. Airapetian et al. [HERMES Collaboration], Phys. Lett. B 693, $11(2010)$

29. E.S. Ageev et al. [COMPASS Collaboration], Nucl. Phys. B 765, 31 (2007)

30. M. Alekseev et al. [COMPASS Collaboration], Phys. Lett. B 673, 127 (2009)

31. M.G. Alekseev et al. [COMPASS Collaboration], Phys. Lett. B 692, 240 (2010a)

32. C. Adolph et al. [COMPASS Collaboration], Phys. Lett. B 717, 376 (2012)

33. C. Adolph et al. [COMPASS Collaboration], Phys. Lett. B 713, $10(2012)$

34. H. Avakian et al. [CLAS Collaboration], Phys. Rev. Lett. 105, 262002 (2010)

35. X. Qian et al. [Jefferson Lab Hall A Collaboration], Phys. Rev. Lett. 107, 072003 (2011)

36. K. Allada et al. [Jefferson Lab Hall A Collaboration], Phys. Rev. C 89(4), 042201 (2014)

37. Y.X. Zhao et al. [Jefferson Lab Hall A Collaboration], Phys. Rev. C 90(5), 055201 (2014)

38. P. Zavada, Phys. Rev. D 55, 4290 (1997)

39. P. Zavada, Phys. Rev. D 65, 054040 (2002)

40. P. Zavada, Phys. Rev. D 67, 014019 (2003)

41. P. Zavada, Eur. Phys. J. C 52, 121 (2007)

42. A.V. Efremov, O.V. Teryaev, P. Zavada, Phys. Rev. D 70, 054018 (2004)

43. A.V. Efremov, P. Schweitzer, O.V. Teryaev, P. Zavada, Phys. Rev. D 80, 014021 (2009)

44. P. Zavada, Phys. Rev. D 83, 014022 (2011)

45. A.V. Efremov, P. Schweitzer, O.V. Teryaev, P. Zavada, Phys. Rev. D 83, 054025 (2011)

46. H. Avakian, A.V. Efremov, P. Schweitzer, F. Yuan, Phys. Rev. D 81, 074035 (2010)

47. U. D' Alesio, E. Leader, F. Murgia, Phys. Rev. D 81, 036010 (2010)

48. F. James, M. Roos, Comput. Phys. Commun. 10, 343 (1975) 
49. F. James, M. Winkler, Minuit User's Guide: C++ Version (2004)

50. J. Pumplin, D. Stump, R. Brock, D. Casey, J. Huston, J. Kalk, H.L. Lai, W.K. Tung, Phys. Rev. D 65, 014013 (2001)

51. D. de Florian, O.A. Sampayo, R. Sassot, Phys. Rev. D 57, 5803 (1998)

52. D. de Florian, R. Sassot, Phys. Rev. D 62, 094025 (2000)

53. L.E. Gordon, M. Goshtasbpour, G.P. Ramsey, Phys. Rev. D 58, 094017 (1998)

54. E. Leader, A.V. Sidorov, D.B. Stamenov, Phys. Lett. B 462, 189 (1999)

55. E. Leader, A.V. Sidorov, D.B. Stamenov, Phys. Lett. B 445, 232 (1998a)

56. E. Leader, A.V. Sidorov, D.B. Stamenov, Int. J. Mod. Phys. A 13, $5573(1998 b)$

57. J. Blumlein, H. Bottcher, Nucl. Phys. B 636, 225 (2002)

58. Y. Goto et al. [Asymmetry Analysis Collaboration], Phys. Rev. D 62, 034017 (2000)

59. D. de Florian, R. Sassot, M. Stratmann, W. Vogelsang, Phys. Rev. Lett. 101, 072001 (2008)

60. M. Hirai et al. [Asymmetry Analysis Collaboration], Nucl. Phys. B 813, 106 (2009)

61. J. Blumlein, H. Bottcher, Nucl. Phys. B 841, 205 (2010)

62. D. de Florian, R. Sassot, M. Stratmann, W. Vogelsang, Phys. Rev. D 80, 034030 (2009)

63. E. Leader, A.V. Sidorov, D.B. Stamenov, Phys. Rev. D 80, 054026 (2009)

64. S. Atashbar Tehrani, F. Taghavi-Shahri, A. Mirjalili, M.M. Yazdanpanah, Phys. Rev. D 87(11), 114012 (2013). Erratum: [Phys. Rev. D 88(3), 039902 (2013)]

65. F. Taghavi-Shahri, H. Khanpour, S. Atashbar Tehrani, Z. Alizadeh Yazdi, Phys. Rev. D 93(11), 114024 (2016)

66. H. Khanpour, S.T. Monfared, S. Atashbar Tehrani, Phys. Rev. D 95(7), 074006 (2017)

67. M. Tanabashi et al. [Particle Data Group], Phys. Rev. D 98(3), 030001 (2018)

68. B. Lampe, E. Reya, Phys. Rep. 332, 1 (2000)

69. M. Lacombe, B. Loiseau, R. Vinh Mau, J. Cote, P. Pires R. de Tourreil, Phys. Lett. 101B, 139 (1981)

70. W.W. Buck, F. Gross, Phys. Rev. D 20, 2361 (1979)

71. M.J. Zuilhof, J.A. Tjon, Phys. Rev. C 22, 2369 (1980)

72. R. Machleidt, K. Holinde, C. Elster, Phys. Rep. 149, 1 (1987)

73. A.Y. Umnikov, L.P. Kaptari, K.Y. Kazakov, F.C. Khanna, AIP Conf. Proc. 339, 79 (1995)

74. A.N. Khorramian, S. Atashbar Tehrani, S. Taheri Monfared, F. Arbabifar, F.I. Olness, Phys. Rev. D 83, 054017 (2011)

75. A.N. Khorramian, H. Khanpour, S. Atashbar Tehrani, Phys. Rev. D 81, 014013 (2010)

76. S.M. Moosavi Nejad, H. Khanpour, S. Atashbar Tehrani, M. Mahdavi, Phys. Rev. C 94(4), 045201 (2016)

77. H. Khanpour, A. Mirjalili S. Atashbar Tehrani, Phys. Rev. C 95(3), 035201 (2017)

78. H. Khanpour, S.T. Monfared, S. Atashbar Tehrani, Phys. Rev. D 96(7), 074037 (2017)

79. L. Ghasemzadeh, A. Mirjalili, S. Atashbar Tehrani, Phys. Rev. D 100, no. 11, 114017 (2019)

80. C. Ayala, S.V. Mikhailov, Phys. Rev. D 92(1), 014028 (2015)

81. I.S. Barker, B.R. Martin, G. Shaw, Z. Phys. C 19, 147 (1983)

82. I.S. Barker, B.R. Martin, Z. Phys. C 24, 255 (1984)

83. V.G. Krivokhizhin, S.P. Kurlovich, V.V. Sanadze, I.A. Savin, A.V. Sidorov, N.B. Skachkov, Z. Phys. C 36, 51 (1987)

84. V.G. Krivokhizhin, S.P. Kurlovich, R. Lednicky, S. Nemecek, V.V. Sanadze, I.A. Savin, A.V. Sidorov, N.B. Skachkov, Z. Phys. C 48, 347 (1990)

85. J. Chyla, J. Rames, Z. Phys. C 31, 151 (1986)

86. I.S. Barker, C.S. Langensiepen, G. Shaw, Nucl. Phys. B 186, 61 (1981)
87. A.L. Kataev, A.V. Kotikov, G. Parente, A.V. Sidorov, Phys. Lett. B 417, 374 (1998)

88. S.I. Alekhin, A.L. Kataev, Phys. Lett. B 452, 402 (1999)

89. A.L. Kataev, G. Parente, A.V. Sidorov, Nucl. Phys. B 573, 405 (2000)

90. A.L. Kataev, G. Parente, A.V. Sidorov, Fiz. Elem. Chast. Atom Yadra 34, 43 (2003). [Phys. Part. Nucl. 38(6), 827 (2007)]

91. A.L. Kataev, Pisma Zh. Eksp. Teor. Fiz. 81, 744 (2005)

92. A. Vogt, Comput. Phys. Commun. 170, 65 (2005)

93. D. Tan, Z. Chen, J. Math. Sci. Math. Educ. 7, 1 (2012)

94. K. Abe et al. [E143 Collaboration], Phys. Rev. D 58, 112003 (1998)

95. A. Airapetian et al. [HERMES Collaboration], Phys. Lett. B 442, 484 (1998)

96. A. Airapetian et al. [HERMES Collaboration], Phys. Rev. D 75, 012007 (2007)

97. J. Ashman et al. [European Muon Collaboration], Phys. Lett. B 206, 364 (1988)

98. P.L. Anthony et al. [E155 Collaboration], Phys. Lett. B 493, 19 (2000)

99. B. Adeva et al. [Spin Muon Collaboration], Phys. Rev. D 58, 112001 (1998)

100. M.G. Alekseev et al. [COMPASS Collaboration], Phys. Lett. B 690, 466 (2010b)

101. V.Y. Alexakhin et al. [COMPASS Collaboration], Phys. Lett. B 647, 8 (2007a)

102. C. Adolph et al. [COMPASS Collaboration], Phys. Lett. B 753, 18 (2016)

103. K.V. Dharmawardane et al. [CLAS Collaboration], Phys. Lett. B 641, 11 (2006)

104. R. Fersch et al. [CLAS Collaboration], Phys. Rev. C 96(6), 065208 (2017)

105. P.L. Anthony et al. [E142 Collaboration], Phys. Rev. D 54, 6620 (1996)

106. K. Ackerstaff et al. [HERMES Collaboration], Phys. Lett. B 404, 383 (1997)

107. K. Abe et al. [E154 Collaboration], Phys. Rev. Lett. 79, 26 (1997)

108. K.M. Kramer [Jefferson Lab E97-103 Collaboration], AIP Conf. Proc. 675, 615 (2003)

109. X. Zheng et al. [Jefferson Lab Hall A Collaboration], Phys. Rev. C 70, 065207 (2004)

110. K. Kramer, D.S. Armstrong, T.D. Averett, W. Bertozzi, S. Binet, C. Butuceanu, A. Camsonne, G.D. Cates et al., Phys. Rev. Lett. 95, $142002(2005)$

111. P.L. Anthony et al. [E155 Collaboration], Phys. Lett. B 463, 339 (1999)

112. E.S. Ageev et al. [COMPASS Collaboration], Phys. Lett. B 612 , 154 (2005)

113. V.Y. Alexakhin et al. [COMPASS Collaboration], Phys. Lett. B 647, 8 (2007)

114. C. Adolph et al. [COMPASS Collaboration], Phys. Lett. B 769, 34 (2017)

115. D. Stump, J. Pumplin, R. Brock, D. Casey, J. Huston, J. Kalk, H.L. Lai, W.K. Tung, Phys. Rev. D 65, 014012 (2001)

116. J. Blumlein, H. Bottcher, A. Guffanti, Nucl. Phys. B 774, 182 (2007)

117. R. Abbate, M. Fickinger, A.H. Hoang, V. Mateu, I.W. Stewart, Phys. Rev. D 83, 074021 (2011)

118. P.A. Zyla et al. [Particle Data Group], PTEP 2020(8), 083C01 (2020)

119. M. Gluck, E. Reya, M. Stratmann, W. Vogelsang, Phys. Rev. D 63, 094005 (2001)

120. E. Leader, A.V. Sidorov, D.B. Stamenov, Phys. Rev. D 73, 034023 (2006) 